\title{
RESEARCH ON THE NETWORK MAP SERVICE TECHNOLOGY OF REMOTE SENSING IMAGE INTELLIGENT CONVERSION BASED ON GAN MODEL
}

\author{
Dejin Tang ${ }^{1}$, Wei Huang ${ }^{1}$, Zhuhua Zha ${ }^{1}$, Jing Yang ${ }^{1}$, Xiaoming Zhou ${ }^{2}{ }^{*}$,Cong Wang ${ }^{1}$ \\ ${ }^{1}$ National Geomatics Center of China, 100830 Beijing,China - (tangdejin, huangwei, zhazh, yangjing,wangcong)@ngcc.cn \\ ${ }^{2}$ Beijing Institute of Remote Sensing Information,100085 Beijing,China - zxm2913@163.com
}

Commission III, WG III/6

KEY WORDS: GAN(Generative Adversarial Networks), Remote sensing image, Intelligent conversion, The network map.

\begin{abstract}
:
Based on an improved generative adversarial networks algorithm (CGAN), this paper explores a technical way to realize map transformation through autonomous learning and training of remote sensing images. Just skip the trial process vector data update and cumbersome process of mapping the basic map elements can be automatically transform, the image on the main streets and typical rules of construction material, can achieve automatic identification and transformation, greatly shorten the tile map production and update cycle, improve the efficiency of the network map service quality, The results of the test platform have proved that it can be applied to a certain extent and can basically meet the requirements of network map production.
\end{abstract}

\section{INTRODUCTION}

\subsection{General Instructions}

Providing map service based on network has become a mainstream way of geospatial data application. From a strategic point of view, government agencies are also actively promoting national network mapping services. At present, tiled map production and update methods is mainly based on remote sensing image. In this way, image recognition and extraction are used to update vector data to complete mapping, and tile maps are generated to provide services. This method has the advantages of long production cycle, high cost, large amount of engineering and complex technology, especially for the generation of multi-scale and multi-resolution tile map.Generative adversarial networks (GAN) are one of the most promising methods for unsupervised learning in complex distribution of deep learning in recent years. GAN generate excellent output through game learning between generators and discriminators, and have achieved good applications in image generation, enhancement and style transformation, etc. The intelligent transformation tile map model of remote sensing image based on generative adversarial network will directly skip the vector data extraction and tedious mapping process, greatly shorten the tile map production and update cycle, improve the timeliness and service quality of network map service, and meet the timeliness requirements of network map service application. this paper explores a technical way to realize map transformation through autonomous learning and training of remote sensing images. Just skip the trial process vector data update and cumbersome process of mapping the basic map elements can be automatically transform, the image on the main streets and typical rules of construction material, can achieve automatic identification and transformation, greatly shorten the tile map production and update cycle, improve the efficiency of the network map service quality, The results of the test platform have proved that it can be applied to a certain extent and can basically meet the requirements of network map production.

\section{MAIN TECHNOLOGIES OF THE NETWORK MAP SERVICE PLATFORM}

At present, remote sensing images and network map services have become important supports for daily work. How to transform remote sensing image into network map quickly and accurately has important practical significance and application value. In recent years, the research of remote sensing intelligent network map conversion using generative adversarial networks (GAN) has been carried out abroad. Generative adversarial networks (GAN) are one of the most promising methods for unsupervised learning in complex distribution in deep learning in recent years. GAN generate excellent output through game learning between generators and discriminators, and have achieved good applications in image generation, enhancement and style transformation, etc. The remote sensing image can be directly transformed into tile map based on generative adconfrontation network, and the production and update cycle of tile map can be greatly shortened by skipping vector data extraction, update and tedious mapping directly.

Based on samples of network map service platform on-line automatic remote sensing image building technology and the network map samples to libraries, to train the map of remote sensing image intelligent switching network model based on GAN, used to build the model of remote sensing image to Internet map service fast intelligent transformation, combined with the actual demand, the special elements such as text notes, point symbol generation technique, in order to form for users to use the elements of a complete network map, at the same time in order to satisfy different users demand for different levels of the network map, network at different levels to map processing, consistency and network map fidelity of the remote sensing

Corresponding author 
image similarity calculation and evaluation, To ensure the accuracy and availability of conversion results. The overall research idea is shown in the figure below:

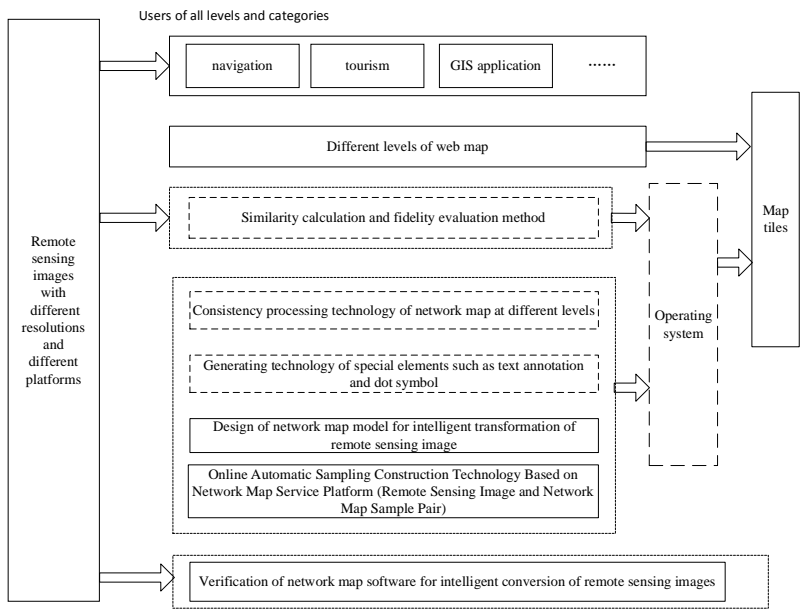

Figure 1. Overall technology roadmap.

\section{DESIGN OF NETWORK MAP MODEL FOR INTELLIGENT CONVERSION OF REMOTE SENSING IMAGES}

\subsection{GAN-based remote sensing intelligent conversion network map research}

Generative Adversarial Networks (GAN) are one of the most promising methods for unsupervised learning in complex distribution in deep learning in recent years. GANs generate excellent output through game learning between generators and discriminators, and have achieved good applications in image generation, enhancement and style transformation, etc.

Generative Adversarial Networks (GAN) proposed by Goodfellow et al is a Generative model, whose main idea is: inspired by the two-person zero-sum game in game theory in structure (namely, the sum of the interests of two people is zero, and what one party gains is what the other party loses), it is composed of a generator $G$ and a discriminator D. G Capture the mathematical distribution model of real data samples, and generate new data samples from the learned distribution model; $\mathrm{D}$ is a binary classifier used to determine whether the input is real data or generated samples. The two methods continue to learn, improve their respective generating ability and discriminant ability, and have significant improvement in enhancing the diversity of generated samples compared with the traditional methods.

At present, there are many autoencoders and Variational autoencoders combining GAN, aiming to improve the authenticity and diversity of GAN generated images.

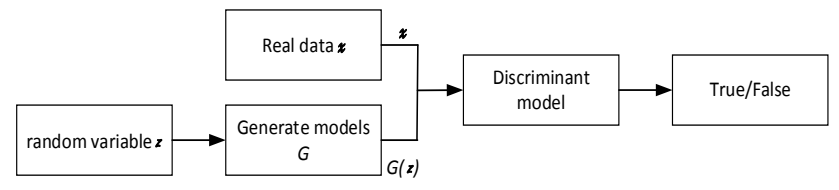

Figure 2. GAN structure.

The structure of GAN is shown in the figure above. D and G represent discriminator and generator respectively, and their structures are both CNN. The input of D is real data X, and the output is 1 or 0 ; The input of $\mathrm{G}$ is a one-dimensional random noise vector $\mathrm{z}$, and the output is $\mathrm{G}(\mathrm{z})$. The goal of training is to make the distribution of $\mathrm{G}(\mathrm{Z})$ as close as possible to the distribution of real data pdata. The goal of $D$ is to realize the binary classification of the input data. If the input comes from the real sample, the output of $D$ is 1 . If the input is $G(z)$, then the output of $D$ is 0 . The goal of $G$ is to make the performance $\mathrm{D}(\mathrm{G}(\mathrm{z}))$ of the data $\mathrm{G}(\mathrm{z})$ generated by $\mathrm{G}$ and the performance $\mathrm{D}(\mathrm{x})$ of the real data $\mathrm{X}$ on $\mathrm{D}$ as consistent as possible. The loss function of $\mathrm{G}$ is calculated according to Equation (1) :

$\operatorname{minVG}(\mathrm{D}, \mathrm{G})=\min (\mathrm{Ez} \sim \operatorname{pz}(\ln (1-\mathrm{D}(\mathrm{G}(\mathrm{z})))))$

In the process of constant antagonistic learning of $\mathrm{G}$, the generated data $G(z)$ is more and more close to the real sample, and the discrimination of $D$ on $G(z)$ is more and more fuzzy. The loss function of D is calculated according to Equation (2) :

$\max \operatorname{VD}(\mathrm{D}, \mathrm{G})=\max (\mathrm{Ex} \sim \operatorname{pdata}(\ln \mathrm{D}(\mathrm{x}))+\mathrm{Ez} \sim \operatorname{pz}(\ln (1-$ $\mathrm{D}(\mathrm{z}))))$

To sum up, the overall loss functions of $G$ and $D$ can be described as shown in Equation (3) :

$\min \mathrm{G} \max \mathrm{D} V(\mathrm{D}, \mathrm{G})=\min \mathrm{G} \max \mathrm{D}(\mathrm{Ex} \sim \operatorname{pdata}(\ln \mathrm{D}(\mathrm{x}))$ $+\mathrm{Ez} \sim \mathrm{pz}(\ln (1-\mathrm{D}(\mathrm{z}))))$

\subsection{Improved network map transformation technology of GAN model}

Traditional GAN models can only learn one class of data at a time. For data sample sets containing multiple classes, it is necessary to learn and generate enhanced sample sets of corresponding classes class by class. Therefore, inefficiency is the main drawback of the model. Mirza et al. proposed the Conditional Generative Adversarial Network (CGAN) model, which is a conditionally controlled GAN. By adding the same Conditional Y(such as the label of data) to the generator and discriminator, Thus, the control condition of GAN model is realized. Compared with the traditional GAN, the total loss function of the former is modified in the CGAN model. The new total loss function is shown in Equation (4) :

$\min \mathrm{G} \max \mathrm{D} V(\mathrm{D}, \mathrm{G})=\min \mathrm{G} \max \mathrm{D}(\mathrm{Ex} \sim \operatorname{pdata}(\ln \mathrm{D}(\mathrm{x} \mid \mathrm{Y}))$ $+\mathrm{Ez} \sim \mathrm{pz}(\ln (1-\mathrm{D}(\mathrm{z} \mid \mathrm{Y}))))$

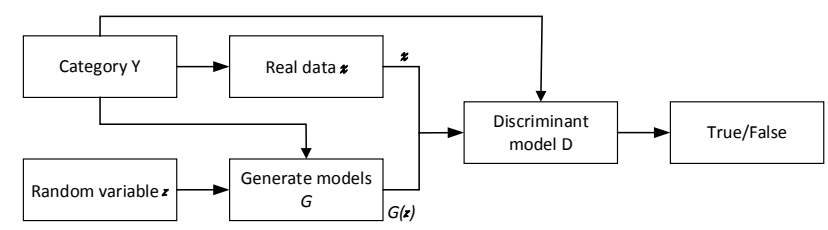

Figure 3. CGAN loss function 


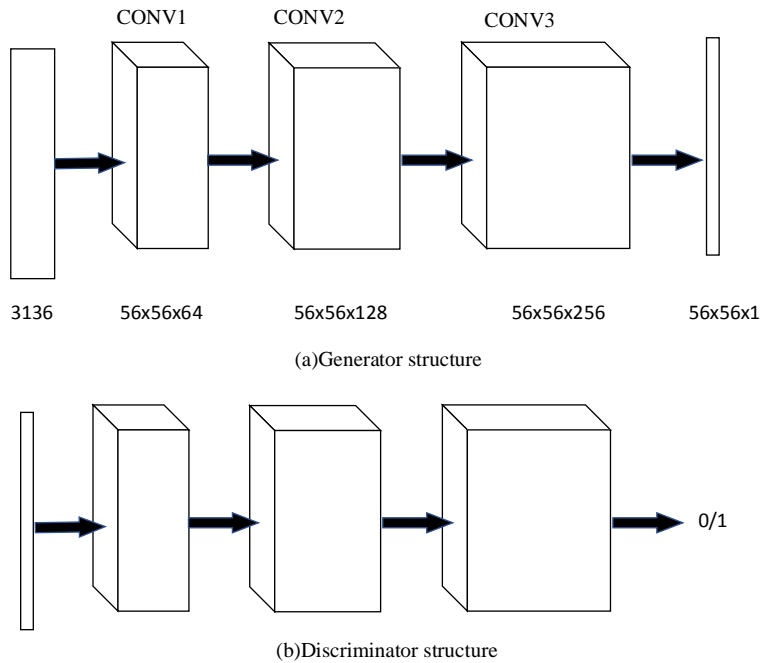

Figure 4. CGAN network structure.

The network map model of remote sensing image transformation is a typical CGAN structure, but $\mathrm{G}$ only accepts a fixed input $\mathrm{X}$, which can be understood as a condition $\mathrm{C}$, that is, no random noise is required, and then outputs the transformed image $\mathrm{Y}$. D accepts an $\mathrm{X}(\mathrm{C}$ in CGAN) and a $\mathrm{Y}$ (true or false sample), and determines whether $\mathrm{X}$ and $\mathrm{Y}$ are matched. In the process of training, $G$ produces images that $D$ cannot distinguish between true and false, and $\mathrm{D}$ tries its best to detect the "forged" images of G.
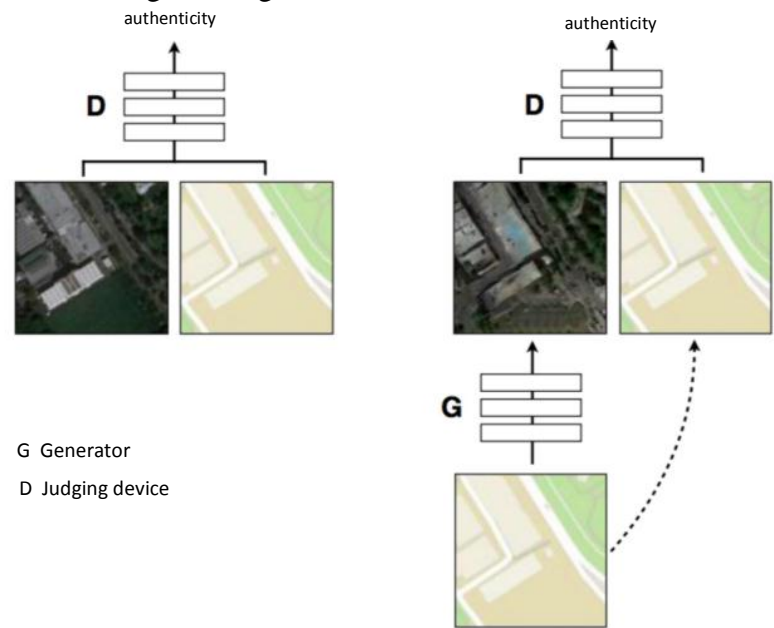

Figure 5. Training process of remote sensing image conversion network map.

\section{ONLINE AUTOMATIC CONSTRUCTION OF SAMPLES BASED ON NETWORK MAP SERVICE PLATFORM}

The data structure of the network Map service platform is layered tile structure. Taking Google Map as an example, Google satellite Map divides images of different resolutions into 20 layers to display. Each level is divided into $256 \times 256$ pixel images in JPG compression format. When the user requests to browse, the server only transmits some images needed in a certain layer of computer display area, generally in 16 to $20256 \times 256$ pixel images, thus greatly reducing the data needed to transmit.
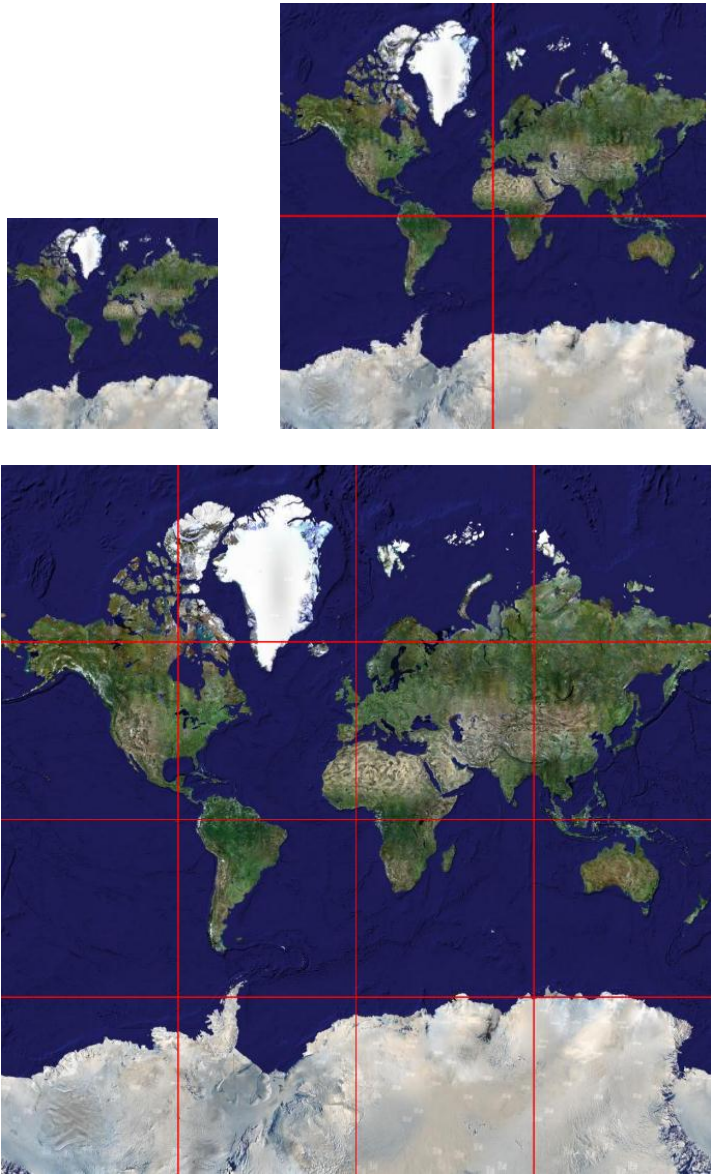

Figure 6. Layer-by-layer segmentation of GM satellite map

The online automatic sample construction technology based on network map service platform mainly studies the methods of obtaining training samples of remote sensing images and sagegrid maps at different levels based on different open network map service platforms, mainly including sample selection rules, sample automatic acquisition, sample database construction management, etc. The process is shown in the figure below:

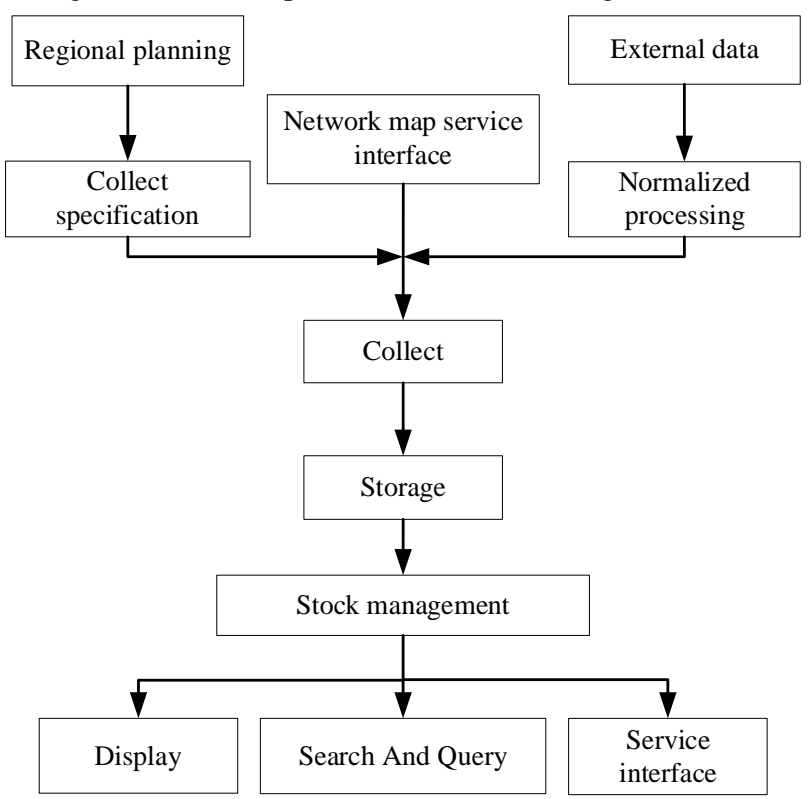

Figure 7. Technical approach to sample construction. 


\subsection{Formulate sample selection rules}

In order to meet the requirements of generating training samples of countermeasures network, the sample selection should be analyzed and determined in accordance with the following principles:

- Sample size and shape $(256 \times 256 ; 512 \times 512)$; It needs to be determined according to model research test;

- Different online map service platforms;

- Different levels and resolutions (different levels represent different resolutions for remote sensing images and different scales for network maps);

- Different seasons (different latitudes, lush vegetation, fallen leaves, snow and ice cover);

- Different landforms and landforms (high mountain, mountain hill, plain, desert, snow mountain, grassland, forest, farmland, water area, city, country);

\subsection{Planning and design of regional sample collection}

For the selected area, coordinate sequence files of sample slices are automatically obtained according to different levels, sample sizes and interval distances.

According to the overall task planning of the construction of the sample slice database in the selected area, the overall planning and design of the collected sample slice area are completed, and the design results can be visually browsed, edited and modified. At the same time, the planning and design results can be saved and loaded. Specific functions include:

- Management and visualization of global underlying data

- Planning and design of sample pieces

- The sample area has been collected for loading display

- Visual display of design results

- Edit and modify the design results

- Division of sample sample collection area

- Preservation of design results

\subsection{Online automatic acquisition of samples}

The sample collection/monitoring adopts the technical implementation scheme of multi-user simulation and user browsing behavior simulation, in order to meet the service rules of Google server and take the purpose of continuous collection/monitoring. The technical implementation of parallel multipoint Internet access is composed of a number of broadband Internet access lines with independent IP addresses. Its function is to provide a large enough Internet access bandwidth to meet the requirements of collecting large amounts of Google satellite image data. Each acquisition line and module can independently collect the specified data range ergodically, and continuously receive the system's new acquisition range instructions from the acquisition management server for uninterrupted operation. The data collected by each independent acquisition server is summarized into a complete image database in a large area under the control of the acquisition management server.

\subsection{Sample storage and database building management}

To establish a mature and reliable sample database of multi-type remote sensing images and network map data resources is the basis of realizing the map service of intelligent conversion of space reconnaissance remote sensing images. In order to improve the reliability of remote sensing image conversion into network map, it is necessary to establish a large number of correct sample databases of remote sensing image and network map data resources to train the neural network and improve the accuracy of the model.

The training sample is a fixed pixel size raster picture, and the image and the vector grid map sample exist in pairs. It has the general description characteristics of raster files, as well as the spatial attribute information and the use information. Including number, description information, coordinate information, precision information, length and width information, band information, etc.

\begin{tabular}{|c|c|c|c|c|c|}
\hline field name & description & type & $\begin{array}{l}\text { Whether } \\
\text { is empty }\end{array}$ & restriction & $\begin{array}{c}\text { default } \\
\text { value }\end{array}$ \\
\hline GCPID & Sample number & Long & no & sole & no \\
\hline GCPINFO & Describes sample slice information & CString & no & & no \\
\hline L_WGS84 & The WGS 84 longitude & double & no & & 0 \\
\hline B_WGS84 & The WGS 84 latitude & double & no & & 0 \\
\hline H_WGS84 & Ellipsoid height of WGS 84 & double & no & & 0 \\
\hline COORDSYSID & The WKT structure of the original coordinate system & Long & no & & 0 \\
\hline COORDSYSNAME & Raw coordinate system name string & Cstring & no & & no \\
\hline HEIGHTSYSID & EPSG coding of the original elevation system & long & no & & 0 \\
\hline HEIGHTSYSNAME & The original elevation system name string & Cstring & no & & 0 \\
\hline $\mathrm{X}$ & East (longitude) coordinates & double & no & & 0 \\
\hline $\mathrm{Y}$ & Northward (latitude) coordinates & double & no & & 0 \\
\hline $\mathrm{Z}$ & Elevation & double & no & & 0 \\
\hline MXY & Plane accuracy of sample slice & double & no & & 0 \\
\hline $\mathrm{MZ}$ & Elevation accuracy of sample slice & double & no & & 0 \\
\hline IMAGESOURCE & Sample source & CString & no & & no \\
\hline
\end{tabular}




\begin{tabular}{|c|c|c|c|c|c|}
\hline L_BM & $\begin{array}{l}\text { Longitude coordinates of the midpoint at the bottom } \\
\text { edge of the image block }\end{array}$ & double & no & & 0 \\
\hline B_BM & $\begin{array}{l}\text { Latitude coordinates of the midpoint at the bottom of } \\
\text { the image block }\end{array}$ & double & no & & 0 \\
\hline IMGWIDTH & Image block width of sample slice & long & no & $>=11$ & 257 \\
\hline IMGHEIGHT & Sample image block height & long & no & $>=11$ & 257 \\
\hline BANDCOUNT & The number of bands in the image block & long & no & 1 or 3 & 1 \\
\hline LOWZOOMLEVEL & Minimization of low resolution images & long & no & 0or $>=2$ & 0 \\
\hline IMGBITS & An image block centered on the sample slice & BLOB & no & $<1 \mathrm{MB}$ & no \\
\hline USEDCOUNT & The number of times the sample was used & long & no & $>=11$ & 0 \\
\hline
\end{tabular}

Table 1 Table of sample basic information

\section{TEST VERIFICATION}

The above technological achievements are utilized to develop an experimental verification platform. High-resolution (1M resolution) remote sensing images are adopted in combination with CGAN model learning technology to complete the automatic transformation of maps and realize network platform services. The results are shown in the figure below.
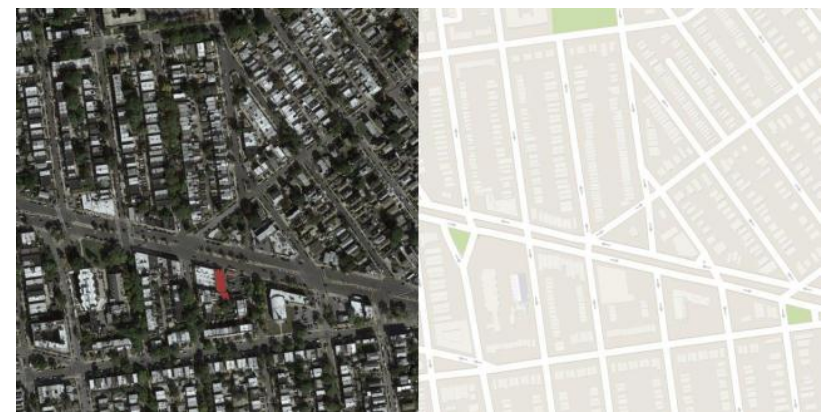

Figure 8. Remote sensing image conversion network map.

As can be seen from the above figure, the technical route in this paper can realize the automatic conversion of basic map elements, and can automatically identify and transform the main streets and typical regular buildings in the image map, which has a certain value of popularization and application.

\section{SUMMARIZES}

Based on an improved generative adversarial network algorithm (CGAN), this paper explores a technical way to realize map transformation through autonomous learning and training of remote sensing images. The test process directly skipped the vector data extraction and update and the tedious mapping process, greatly shortened the tile map production and update cycle, and improved the timeliness and service quality of network map service. The test platform results have proved that it has a certain application and can basically meet the requirements of network map production.

\section{ACKNOWLEDGEMENTS}

This work was supported by the National Key Research and Development Program of China (NO.2017YFB0503705)

\section{REFERENCES}

A. Radford, L. Metz, S. Chintala, 2016. Unsupervised representation learning with deep convolutional generative adversarial networks[C]. International Conference on Learning Representations, San Juan, 187-199

A.VandenOord,N.Kalchbrenner,L.Espeholt,2016.Conditional image generation with pixel cnn decoders[C]. International Conference on Neural Information Processing Systems, Barcelona,4790-4798.

A.Odena, C. Olah, J. Shlens, 2017.Conditional Image Synthesis With Auxiliary Classifier GANs[C].International Conference On Machine Learning, Sydney, 2642-2651.

Brahim T., Bouazra A., Said M.,2021.Numerical modeling of linear and nonlinear optical properties in hexagonal core-shell shape $\mathrm{ZnSe} / \mathrm{ZnSxSe} 1-\mathrm{x}$ quantum dot[J] Optik, 225.

D. Li, D. Chen, B. Jin, et al, 2019. Mad-gan: Multivariate anomaly detection for time series data with generative adversarial networks[C]. International Conference on Artificial Neural Networks, Mu-nich, 703-716

D. Berthelot, T. Schumm, L. Metz. Began, 2017. Boundary equilibrium generative adversarial networks[J]. arXiv preprint arXiv:1703.10717.

E. L. Denton, S. Chintala, A. Szlam, et al, 2015. Deep generative image models using a laplacian pyramid of adversarial networks[C]. International Conference on Neural Information Processing Systems, Montreal, 1486-1494

F. Juefei-Xu, V. N. Boddeti, M. Savvides, 2017. Gang of gans: Generative adversarial networks with maximum margin ranking[J]. arXiv preprint arXiv:1704.04865.

Goodfellow I., Mirza M., Courville A., Bengio Y, 2013. Multiprediction deep boltzmann machines. Advances in Neural Information Processing Systems, 548-556.

Mirza M, Osindero S,2014. Conditional Generative Adversarial Nets[J]. Computer Science, 2672-2680. 
Mahya Motevalian, Fahimeh Ghavamipour,2020.Bahman Maroufi et al.Mutual effects of protein corona formation on CdTe quantum dots[J] Analytical Biochemistry.

M. Arjovsky, S. Chintala, L. Bottou, 2017. Wasserstein generative adversarial networks[C]. International Conference on Machine Learning, Sydney, 214-223

O.Mogren, 2016, C-rnn-gan:A continuous recurrent neural network with adversarial training[C].Constructive Machine Learning Workshop at NIPS 2016, Barcelona, 32-38

S. Ma, J. Fu, C. Wen Chen, et al, 2018. Da-gan: Instance-level image translation by deep attention generative adversarial networks $[\mathrm{C}]$. Proceedings of the IEEE Conference on Computer Vision and Pattern Recognition, Salt Lake, 5657-5666

T. Salimans, I. J. Goodfellow, W. Zaremba, et al, 2016. Improved techniques for training gans[C]. International Conference on Neural Information Processing Systems, Barcelona, 2234-2242

Urice N. Tohgha, Alexander M. Watson, Nicholas P. Godman,2020.Tuning the Electrowetting Behavior of Quantum Dot Nanofluids[J] Journal of Colloid And Interface Science.

X.Chen,Y.Duan,R.Houthooft, et al, 2016.Infogan: Interpretable representation learning by information maximizing generative adversarial nets[C].International Conference on Neural Information Processing Systems, Barcelona, 2172-2180

X. Mao, Q. Li, H. Xie, et al, 2017. Least squares generative adversarial networks[C]. Proceedings of the IEEE International Conference on Computer Vision, Venice, 2794-2802 